\title{
Enfermedad Mecanobulosa hereditaria: Análisis clínico histopatológico de dos casos
}

\author{
Drs.: P. Zolezzi R.,' Faúndez V., L. Norambuena M., ${ }^{3}$ C. Prieto W.'
}

\begin{abstract}
Two patients with Hereditary Mechanobullous Disease are presented. Clinical considerations and skin histopathological studies allowed us to consider the first case as a Demolytic Bullous Dermatosis Recessive (scarring type) and the second case as a Junctional Bullous Epidernatusis (nonscarring type). Actual classification and therapy of Hereditary Mechanobullous Disorders are fommented. are commented.
\end{abstract}

Las enfermedades mecanobulosas hereditarias son estados crónicos que se caracterizan por la formación de ampollas ante diversos grados de traumatismos de la piel, siendo las más frecuentes las que se agrupan bajo el témnino de Epidermolisis Bulosa.'

El estudio histopatológico diferencia A) Formas no cicatriciales que comprometen la epidermis y B) Formas cicatriciales que comprometen el dermis, por lo que la denominación de Epidermolisis Bulosa para todas las formas de esta entidad no siempre es correcta. ${ }^{2}$

A) Las formas no cicatriciales comprenden:

1. Epidermolisis Bulosa Simple: Las primeras ampolias aparecen generalmente en el período neonatal, siendo más frecuentes en manos y pies, pero pueden presentarse en cualguier sitio de piel traumatizada. Curan sin cicatrización y rara vez comprometen dientes, uñas o mucosas.

Histopatología: Las anpollas se forman por lisis celular en la capa basal de la epidenmis.

2. Erupcion Ampollosa Recurrente de manos y pies (Enfermedad de Weber-Cockaine): La formación de ampollas se produce generalmente después de fricción de manos y pies y aparecen alrededor de los dos o tres años de edad.

Histopatología: Las ampollas se forman en el estrato espinoso de la epidermis.

3. Epidermolisis Ampollosa de Union (letalis-

\footnotetext{
Instituto de Pediatria. Universidud Austral de Chile.

${ }^{2}$ Servicio de Pediatria. Hospital de Valdivia.

3 instituto de Patología. Universidad Austral de Chile.

*Trabajo leído por la DTa. Cecilia Faúndez V. en el Xlli Congreso $N$ acional de Pediatría en Pucón, Noviembre-1979, como su trabujo de ingreso a la Suciedad Chilena de Pediatria.
}

Herlitz): Es la forma más grave y mortal y se presenta a temprana edad; se caracteriza por erosiones extensas de aparición espontánea o ante traumatismos mínimos en cualquier región de la piel, pero especialmente en piernas $y$ regiones glúteas. Destaca el compromiso de mucosa bucal en forma precoz. Frecuentemente hay distrofia ungueal y pérdida de las uñas. La causa de muerte es generalmente la infección. En los pacientes que sobreviven se observan erosiones esofágicas y malformaciones dentarias.

Histopatología: Estudios con microscopía electrónica han revelado que la lesión asienta en la unión dermoepidérmica en el espacio intermembranoso, entre la membrana plasmática y la lámina basal de la capa de células basales. 1,2

B) Las formas cicatriciales comprenden:

1. Dermatosis Ampollosa Dermolitica Dominante: Las ampollas se caracterizan por presentarse principalmente en extremidades, curan lentamente dejando una cicatriz delgada $y$, a veces, pequeñas placas hipopigmentadas o un queloide. Puede haber desprendimiento $y$ atrofia de uñas. Es frecuente el compromiso de mucosa bucal y raro el de esófago.

2. Dermatosis Ampollosa Dermolitica Recesiva: Sigue en gravedad a la Enfermedad de Herlitz. Se caracteriza por la aparición de ampollas en el periodo neonatal que pueden comprometer toda la piel y las mucosas. La cicatrización es lenta dejando lesiones finas como pergamino, siendo frecuentes las cicatrices retráctiles. Suelen observarse deformidades de manos y pies con formación de revestimientos similares a guantes que unen los dedos. Se asocia con anemia y retraso del crecimiento. Histología: En ambas formas la lesión se ubica 
debajo de la lámina basal. Estudios con microscopia electrónica revelan en la forma dominante un probable defecto primario en las fibrillas de colágeno, encontrándose aun en la piel de aspecto normal alteraciones estructurales y disminución en el número de las fibras colágenas, a diferencia de la forma recesiva en que sólo se altera la piel comprometida.

\section{MATERIAL Y METODOS}

Se analizan dos casos clinicos hospitalizados en el Servicio de Pediatría del Hospital de Valdivia, estudiados con: Hemograma, V.H.S., orina, cultivos de lesiones de piel, orina, deposiciones, sangre, L.C.R., Kahn, Rx. tórax y estudio histopatológíco de piel.

Caso I (F. Clínica 192205): lactante de un año un mes de edad, sexo masculino, peso 7.5 $(x)$ grs. que desde su nacimiento presenta múltiples lesiones ampollares y bulosas en piel y mucosa que tienden a generalizarse con remisiones y exacerbaciones periódicas. Se observan múltiples cicatrices retráctiles, distrofia ungueal y pérdida de unas en manos y pies. (Foto 1). Antecedentes de un hemiano fallecido a consecuencia de lesiones similares en el periodo de recién nacido. El estudio histopatológico de la piel revela: desprendimiento total de la epidermis; a ésta adhiere parte de dermis papilar acentuadamente alterada (Fig. 1).

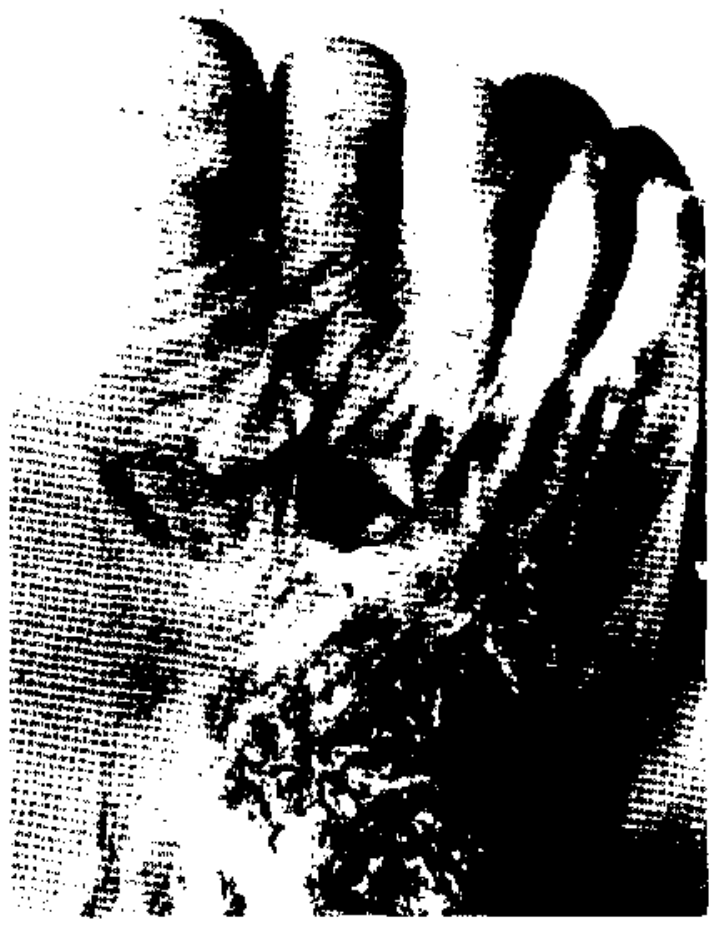

Foto 1: Dennatessis Ampollusia Dennolítica Recesiva: Lesiones cuatriciales y pérdidid de las uñas.

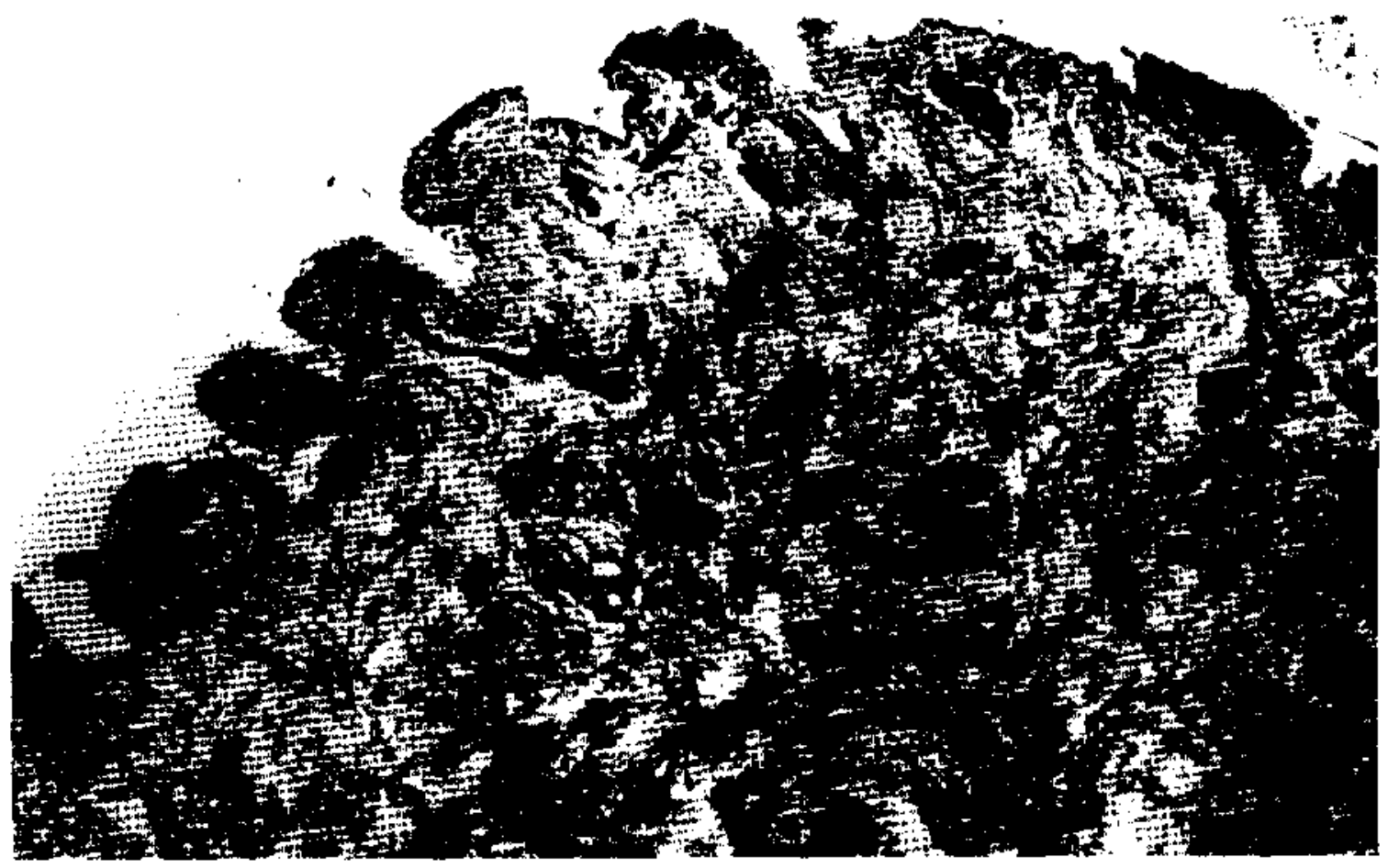

Figure 1: Dematosis Aropollosa Dermolítica Recesiva: Desprendimiento total de la epidermus. No se distingue membrana basal. Colageneu de disposición irregular. 
Cuso 2 (F. Clínica 191018): R.N.T.A.E.G., sexo femenino, peso 2.600 grs. que presenta al nacer lesiones ampollares y bulosis eritematosas y sangrantes en manos, pies y mucosa oral; piel lábil con formación espontánea de lesiones similares en todo el cuerpo, especialmente en las áreas de fricciones, que alcanzan a un $80 \%$ de la superficie corporal. Las lesiones curan sin dejar cicatriz. Posteriormente se agrega distrofia ungueal y pérdida de algunas uñas (Foto 2). Fallece a los 33 días de vida por sepsis, con hemocultivos positivos a Pseudomonas Aeroginosa. El estudio histopatulogico revela piel con extensas ulceraciones que dejan demis desnuda. $A$ este nivel la dermis presenta colonias bacterianas con leve reacción inflamatoria. La epidermis exhibe areas de acantosìs con tendericia a vesiculación de la capa hasal (Fig. 2).

\section{COMENTARIO}

De acuerdo a la clasificación revisada podemos establecer que el caso 1 pertenece a una Dermatosis Ampollosa Dermolítica Recesiva, por la presencia de ampollas y bulas en piel y mucosas desde el nacimiento, que curan con cicatrización apergaminada produciendo contracturas en manos y pies, acompañándose de anemia y desnutri- ción. El informe histopatológico revela compromiso del dermis.

El caso 2 corresponde a una Epidemolisis Bulosa de Unión, por la formación de ampollas y bulas en piel y mucosas desde el nacimiento, que curan sin dejar cicatrización y por el compromiso ungueal. Se diferencia de las otras dos formas no cicatrizantes por ser éstas de curso más benigno, ya que rara vez comprometen mucosas y no se acompañan de alteraciones ungueales. $\mathrm{El}$ informe histopatológico revela el compromiso característico sólo de epidermis.

Con respecto al tratamiento de estas enfermedades aún no se ha logrado encontrar una terapia eficaz. Aislamiento, antisépticos de aplicación tópica $y$ antibióticos por vía general en caso de infección no modifican mayormente el curso mismo de la enfermedad. El uso de corticoides por vía sistémica tampoco ha sido eficaz. La aplicación tópica en solución de glutaraldehído al $10 \%$ parecería ser efectiva en algunas formas de Epidermolisis Bulosa localizada usada en forma continua, ya que reaparecen las lesiones con la suspensión del tratamiento. Ultimamente han apa-

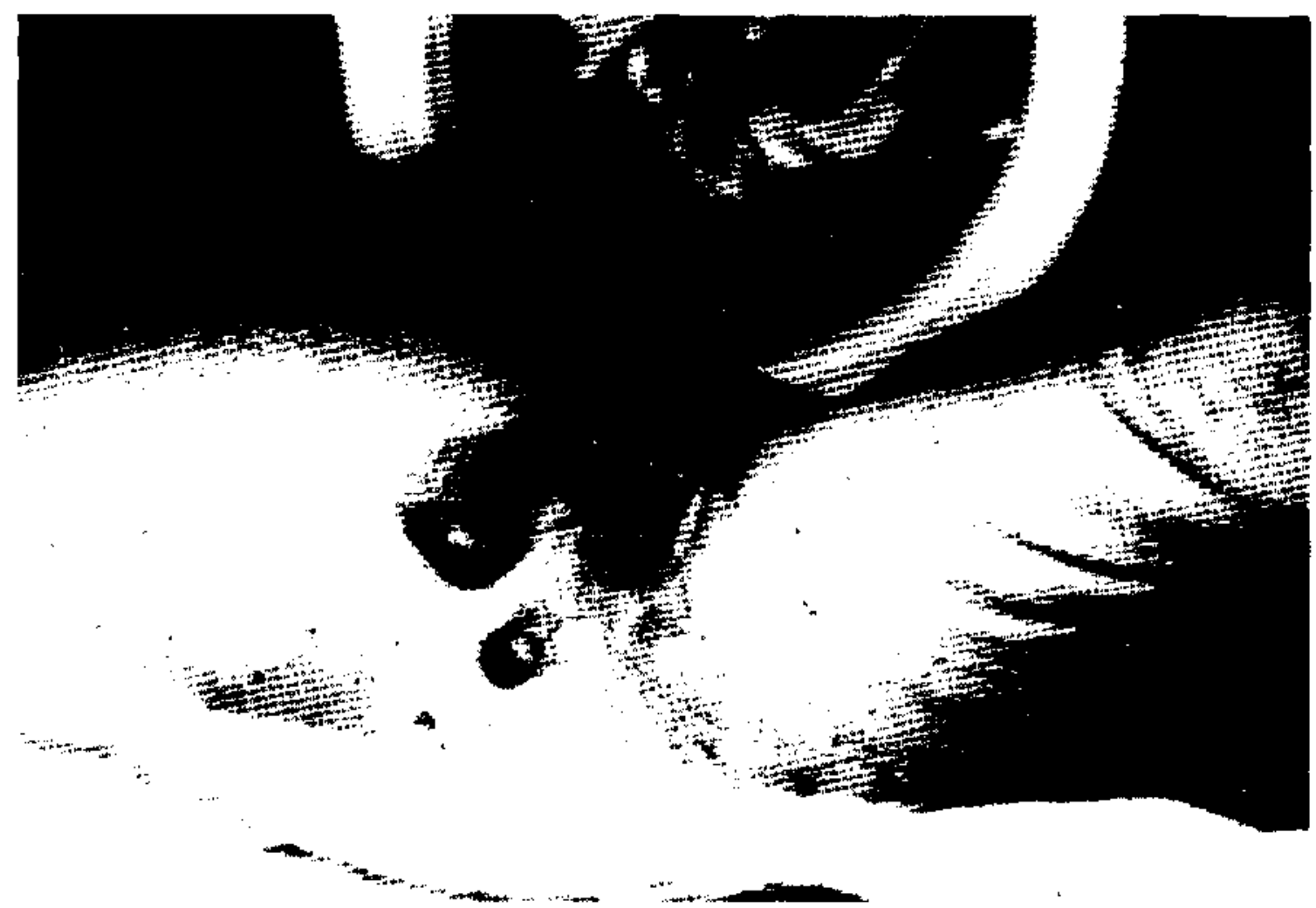

Foto 2: Epidermolisis Bulosa de Union. Lesiones Ampollosas. 


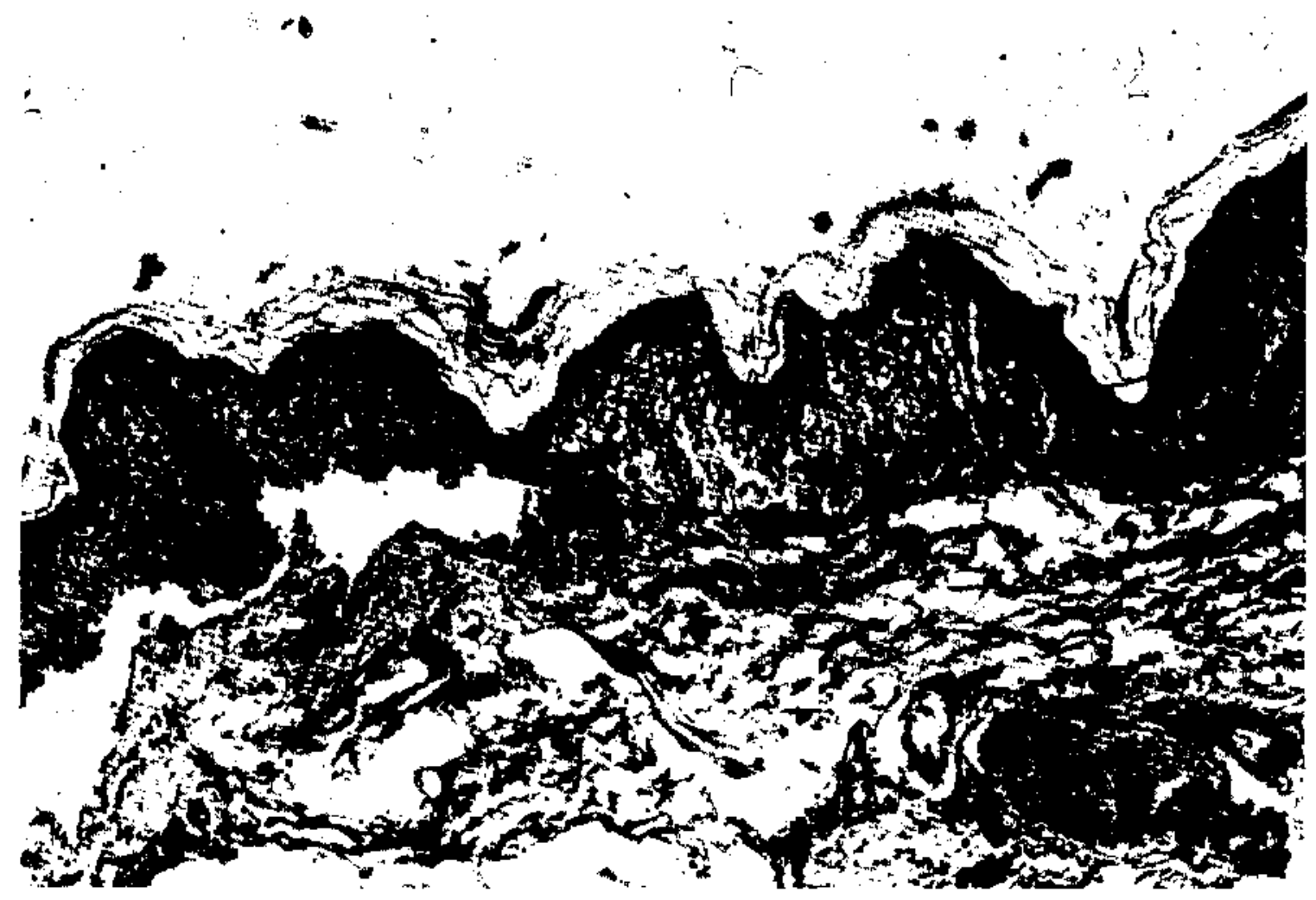

Figura 2: Epidermolisis Bulosa de Unión: Vesicula subepitelial con desprendimiento total de epidemis: Signos incipientes de reepitelización en el borde del fondo de la vesícula.

recido algunas referencias en relación al uso mantenido de vitamina Eः300-600 u. por dia, para los casos de Dermatosis Dermolitica (formas cicatriciales), observándose reaparición de las lesiones al suspender la administración de vitamina E. ${ }^{4}$

\section{RESUMEN}

Se presentan dos casos de Enfermedad Mecanobulosa Hereditaria. Las características clínicas de las lesiones de piel, constituidas fundamentalmente por ampollas y bulas de aparición espontánea junto con el estudio histopatológico de las lesiones de piel permitieron demostrar que el primer caso corresponde a una Dermatosis Ampollar Dermolítica Recesiva (foma cicatricial), y el se- gundo caso a una Epidermolisis Ampollosa de Unión (forma no cicatricia). Se revisan las Enfermedades Mecanobulosas Hereditarias, grupo de enfermedades poco conocidas, comentándose su tratamiento.

\section{REFERENCLAS}

IWatson, W.: "Selected Genodernatoses". Ped. Clin. N. Amer. 25: $263-284$ (1978).

${ }^{2}$ Pearson, R.W., Potter. B.; Strauss, F.: “Epideravolysis Bullosa Hereditaria Letalis. Clinical and histological manifestabons and course of the disease". Arch. Dermatol. 109: 349-355 (1974).

3Le'sGruseilliers, J. Brisson. P.: "Localized Epidermulysis Bullosa. Report of two cuses and evaluation of the rapy with gluta. raldehyde". Arch. Dermatol. 109: 70-72 (1974).

"Hichaeison, J.D.: Schmidt, JD.; Dresden, M.H.; Duncan W.C.: "Vitamin E treatment of Epidenmolysys Bullosa", Arch. Dermatol. 109: 67-69 (1974). 